\title{
The Natural Parallelism
}

\author{
Robert Strzodka \\ Max Planck Institut Informatik, Saarbruecken, Germany
}

\begin{abstract}
With the advent of multi-core processors a new unwanted way of parallel programming is required which is seen as a major challenge. This talk will argue in exactly the opposite direction that our accustomed programming paradigm has been unwanted for years and parallel processing is the natural scheduling and execution model on all levels of hardware.

Sequential processing is a long outdated illusionary software concept and we will expose its artificiality and absurdity with appropriate analogies of everyday life. Multi-core appears as a challenge only when looking at it from the crooked illusion of sequential processing. There are other important aspects such as specialization or data movement, and admittedly large scale parallelism has also some issues which we will discuss. But the main problem is changing our mindset and helping others to do so with better education so that parallelism comes to us as a friend and not enemy.
\end{abstract}

